\title{
A Novel Digital Watermarking Technique based on Feature Attribute Selection using Integer Wavelet Transform Function and ID3 Algorithm
}

\author{
Charu Kavadia \\ M.Tech Scholor \\ Department of Computer Science and Engineering, \\ ACEIT, Jaipur \\ Rajasthan, India
}

\author{
Vishal Shrivastava \\ Associate Professor \\ Department of Computer Science and Engineering, \\ ACEIT, Jaipur \\ Rajasthan, India
}

\begin{abstract}
Feature extraction and classification based digital water marking is new area of research in current privacy protection and copyright technique. In this area of research various authors used feature extraction technique such as wavelet transform function and for classification purpose used support vector machine. in the process of feature based water marking technique feature extraction is most important part and the classification depends on selected feature. In this paper proposed novel digital water marking technique based on integer wavelet transform with attribute based classification technique. For the selection of feature attribute used RBF function. The selection of attribute depends on extracted feature by integer wavelet transform. The proposed method simulates in MATLAB software and tested some reputed attack such as noise attack, share attack and translation attack. our empirical evaluation result shows better performance in compression of DWT water marking technique.
\end{abstract}

\section{Keywords}

Digital watermarking, IWT, ID3 RBF network

\section{INTRODUCTION}

Manipulation of digital water marking is critical issue in current decade of multimedia technology and copyright protection. Manipulation task perform by someone illegal person and unauthorized access of multimedia data. Such behavior changes the ownership of multimedia data. In general Watermarking can be performing into visible and invisible categories. For visible watermarking, the embedded Watermark can be visually observed. That clearly indicates the ownership of the image [5]. The merits of visible watermarking is that it is easy to authenticate the owner of the image without any problem, but its demerits is that the embedded watermark signal can also be easily removed or cracked. An Invisible watermark is intended to be invisible but is detected and extracted by an appropriate piece of tool when the need arises. For the protection of digital water marking various water marking technique are proposed by different reaches. Some are used wavelet domain function for its advantage and some other are used pixel level or bit level watermarking technique. Feature based water marking technique used wavelet transform function for the process of feature extraction. The wavelet transform function is frequency domain function and it is very complex instead of spatial domain function and image embedding technique. Tang and Hang [6] gives a robust digital image watermarking scheme that combines image feature extraction and image normalization is proposed. The goal is to resist both geometric distortion and signal processing attacks. Seo and Yoo [7] proposed a novel method for content-based watermarking based on feature points of an image. At each feature point, the watermark is embedded after scale normalization according to the local characteristic scale. Qi and Qi [8] developed a robust content-based watermarking scheme. This scheme combines the advantages of important feature extraction, Delaunay-tessellation-based triangle matching, perceptual analysis, one-way hash functions, error correcting codes, and spread-spectrum-based blind watermark embedding and retrieval to reduce the watermark synchronization problem and resist geometric distortions and common image processing attacks. Wang et al. [9] proposed a novel feature-based image watermarking scheme against general geometric attacks. This scheme is based upon scale space theory, with the combination of image feature extraction and image normalization. Classification technique also improved the performance of digital water marking technique. in the classification technique used most famous classifier support vector machine are used by various authors. The support vector machine is binary classifier used for classification purpose. The process of classification classified the extracted feature for water mark embedding process. Some authors are used in terms of improved support vector machine and combined with other learning function. $\mathrm{Fu}$ et al. [10] proposed an SVM-based watermarking method in which the difference of the intensity level of pixels of blue components was used to train the SVM. Tsaia et al. [11] presented a robust lossless watermarking algorithm based on $\alpha$-trimmed mean algorithm and support vector machines (SVMs), in which the SVM is trained to memorize the relationship between the watermark and imagedependent watermark other than inserting watermark into the host image. $\mathrm{Li}$ et al. [12] introduced a semi-fragile watermarking algorithm based on SVM"es. This algorithm first gives the definition of wavelet coefficient direction tree, then a relation mathematical model between root node and its offspring nodes is established using SVM and further watermark is embedded and extracted based on this structuring data using relation (relational model). In this paper proposed a novel feature selection based water marking technique based on integer wavelet transform function and attribute based feature selection technique using ID3 algorithm. The IWT (integer wavelet transform) function used as feature extractor and the extracted feature used an attribute for the ID3 classification algorithm. The selection of feature used RBF 
function for embedding process. The above section discuss introduction of water marking technique and feature extraction process. In section II we describe integer wavelet transform function and ID3 algorithm. In section III proposed algorithm. In section IV discuss experimental result analysis. In section and finally conclude in section 5 .

\section{IWT and ID3}

\subsection{IWT}

Integer wavelet transform (IWT) function is great advantage over discrete wavelet transform function. The discrete wavelet transform function gives the fractional value of filter and loss is estimated. but in case of integer wavelet transform function loss are not occurred and encoding task is perform easily. For lossless coding it is necessary to make an invertible mapping from an integer image input to an integer wavelet representation. The lifting scheme is used to construct symmetric biorthogonal wavelet transforms starting from interpolating Deslauriers-Dubuc scaling functions. A family of $(\mathrm{N}, \mathrm{N})$ symmetric biorthogonal wavelets is derived, where $\mathrm{N}$ is the number of vanishing moments of the analysis high-pass filter and $\mathrm{N}$ is the number of vanishing moments of the synthesis high-pass filter. An instance of this family of transforms is the $(4,2)$ interpolating transform. The integer version of it, given in [13], is implemented in the first stage of our coding algorithm. In this case, the integer wavelet representation of a one dimensional signal $\mathrm{A}^{0}(\mathrm{n})$ having $\mathrm{N}$ nonzero samples is given by

$$
\begin{gathered}
\forall n: D^{i+1}(n)=A^{i}(2 n+1)-\left[\sum_{k} p k A^{i}(2(n-k))+\frac{1}{2}\right] \\
0 \leq i<j, 0 \leq n<2^{-(i+1)} N \\
-2 \leq k \leq 1 \\
\forall n: A^{i}(2 n)=A^{i}(2 n)+\left[\sum_{k} u k A D^{i+1}(n-k)+\frac{1}{2}\right] \\
0 \leq i<j, 0 \leq n<2^{-(i+1)} N \\
0 \leq k \leq 1
\end{gathered}
$$

where $[x]$ represents the integer part of $x, j$ is the number of scales, $A^{i+1}(n)$ and $D^{i+1}(n)$ denote, respectively, the Approximation and the detail of the original signal calculated at the scales $(i+1), 0 \leq I<j$. The integer part of transform function gives the better encoding technique. The encoded transform value work as feature for the selection of water marking process.

\subsection{ID3}

ID3 algorithm[16] is a attribute based classification technique in data mining. The flexibility of ID3 algorithm in case of small data is very high. The process of ID3 Algorithm based on information entropy of attribute. in the process of water marking ID3 are used as find common feature for selection process. The ID3 algorithm is the basic algorithm of decision tree induction, it generates decision tree by means of defeating in detail from the top to the bottom.

\author{
Algorithm: \\ The algorithm is as follows: \\ ID3 (data, Target_Attribute, Attributes)
}

- Create a root node for the tree

- If all data are positive, Return the single-node tree Root, with label $=+$.

- If all data are negative, Return the single-node tree Root, with label = -

- If number of predicting attributes is empty, then Return the single node tree Root, with label = most common value of the target attribute in the data.

- Otherwise Begin

$$
\begin{aligned}
& \circ \quad \mathrm{A}=\text { The Attribute that best classifies data. } \\
& \circ \quad \text { Decision Tree attribute for Root }=\mathrm{A} .
\end{aligned}
$$$$
\text { - For each possible value, } v_{i} \text {, of A, }
$$

- Add a new tree branch below Root, corresponding to the test $\mathrm{A}$ $=v_{i}$.

- Let $\operatorname{Data}\left(v_{i}\right)$ be the subset of data that have the value $v_{i}$ for $\mathrm{A}$

- If $\operatorname{Data}\left(v_{i}\right)$ is empty

- Then below this new branch add a leaf node with label $=$ most common target value in the data

- Else below this new branch add the subtree ID3 (Data $\left(v_{i}\right)$ Target_Attribute, Attributes $\{\mathrm{A}\})$

- $\quad$ End

- Return Root

\section{PROPOSED METHODOLOGY}

In this section we discuss the proposed method for digital water marking technique. This technique based on three different functions in single unit, the first part of function is integer wavelet transform function; these function used as feature extractor in 2-D transform. These transform provide two layers of details and approximate. The approximate part process and encode then generates the feature coefficient for attribute selection. The second part of algorithm work as attribute selection for both the image host image and symbol image. The selected attribute in both the image perform RBF function for pattern generation and finally image are embedded.

The process of watermark embedding steps is given below:

1. The size of host image is $512 \times 512$ and the size of water mark is $128 \times 128$.

2. both these image passes through the IWT transform function (2D) and generates feature block matrix

3. the generated matrix are encode

4. the detail part of host image set default parameter of PSNR value for compression operator for selection purpose

5. scan the matrix data feature attribute of host image and water mark image

6. generate feature attribute of each matrix

7. compute the entropy of feature attribute for the root node 


$$
\begin{aligned}
& \text { Entropy }(D) \\
& =-\sum_{i=1}^{N} p i \log p i
\end{aligned}
$$

8. compute the information gain of feature attribute

$$
F B(v)=\sum_{j=1}^{N} P j[p i \log p i]
$$

9. compute the gain of each feature attribute Gain $(v)=E n t r o p y(D)-F B(v)$

10. determine maximum gain equal to PSNR value and split encode feature in Gaussian form

11. apply RBF function for pattern generation

12. embedded process are done

13. exit

The water mark extraction process from a watermarked image are given below

1. apply 2-D IWT transform function on watermark image

2. different encoded feature in random Oder and find entropy of each feature

3. compute gain of maximum PSNR value of feature attribute

4. calculate output of each attribute in gain(v)

5. deform the RBF trained cluster the cluster center value is 0

6. Original watermark is obtained after cluster deformation.

\section{EXPERIMENTAL RESULT ANALYSIS}

The proposed watermarking algorithms are implemented using MATLAB. The imperceptibility and the robustness of the watermarked image are tested with PSNR and NC. Three family images and one Lena image of size $512 \times 512$ is selected as the cover image. Color image of size 128x128 local is selected as the watermark. The PSNR of the watermarked image is calculated using the formula

$$
P S N R=10 \log _{10}\left(\frac{\sum_{i=1}^{N} \sum_{j=1}^{N}(\mathrm{~F}(\mathrm{i}, \mathrm{j}))^{2}}{\sum_{i=1}^{N} \sum_{j=1}^{N}(\mathrm{f}(\mathrm{i}, \mathrm{j})-\mathrm{F}(\mathrm{i}, \mathrm{j}))^{2}}\right)
$$

Where $\mathrm{R}$ is maximum fluctuation in the cover image $=511$

$$
\sum_{j=1}^{M S E} \sum_{k=1}^{c} \frac{\mathrm{w}(\mathrm{j}, \mathrm{k})-\mathrm{w}^{\prime}(\mathrm{j}, \mathrm{k})}{\mathrm{rc}} .
$$

Where

$\mathrm{r}=$ number of rows in the digital image $\mathrm{c}=$ number of columns in digital image $w(j, k)=$ cover image $w(j, k)=$ cover image
$N C=\frac{\sum_{\mathrm{j}} \sum_{\mathrm{k}} \mathrm{W}(\mathrm{j}, \mathrm{k}) * \mathrm{~W}^{\prime}(\mathrm{j}, \mathrm{k})}{\sum_{\mathrm{j}} \sum_{\mathrm{k}} \mathrm{W}(\mathrm{j}, \mathrm{k}) * \mathrm{~W}(\mathrm{j}, \mathrm{k})}$

The performance evaluation of the methods is done by measuring imperceptibility and robustness. The normalized correlation coefficient (NC) is used to measure the similarity between the cover image and the watermarked image. Peak Signal-to-Noise Ratio (PSNR) is used to measure the imperceptibility of the watermarked image. The robustness of the watermarked image is tested by attacks such as JPEG compression, cropping, median filtering, salt \& pepper noise attack, and rotation[13].
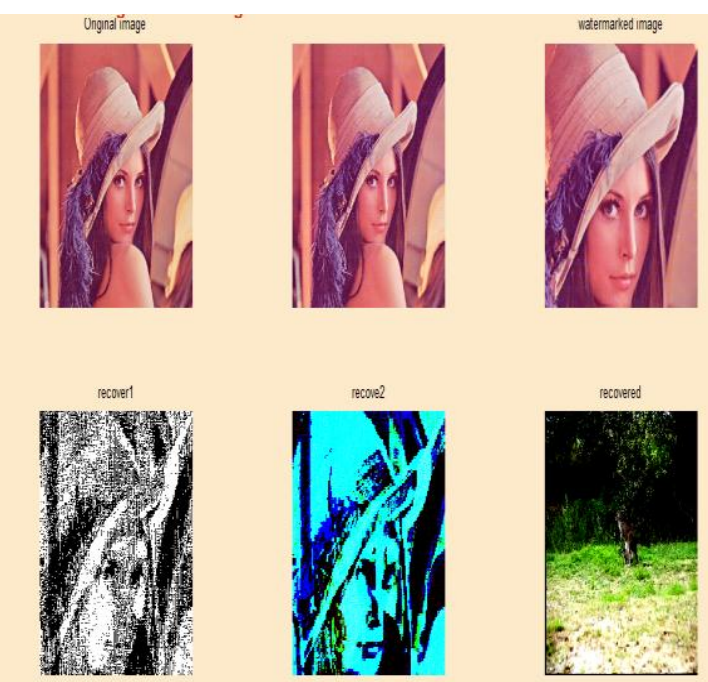

Figure 3 indicated that there were no visual differences between host image and the image embedded Watermark. And the watermarking had good imperceptibility. The peak signal to noise ratio for shear feature is PSNR $=23.05$, the extracted watermark and the original watermarking similarity was $\mathrm{NC}$ $=0.6431$.

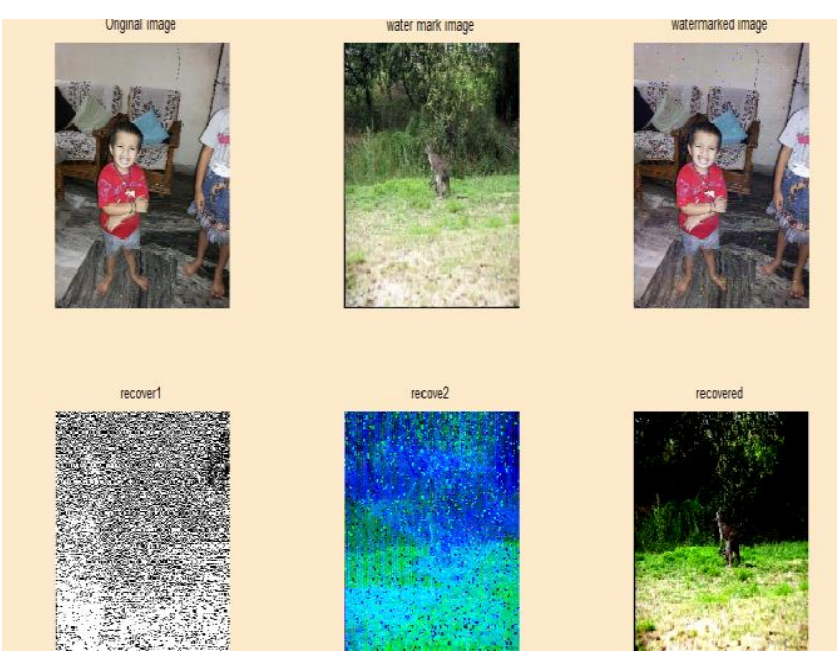

Figure 4 indicated that there were no visual differences between host image and the image embedded Watermark. And the watermarking had good imperceptibility. The peak signal to noise ratio for cropping feature is PSNR $=25.0000$, the extracted watermark and the original watermarking similarity was $\mathrm{NC}=0.3651$. 

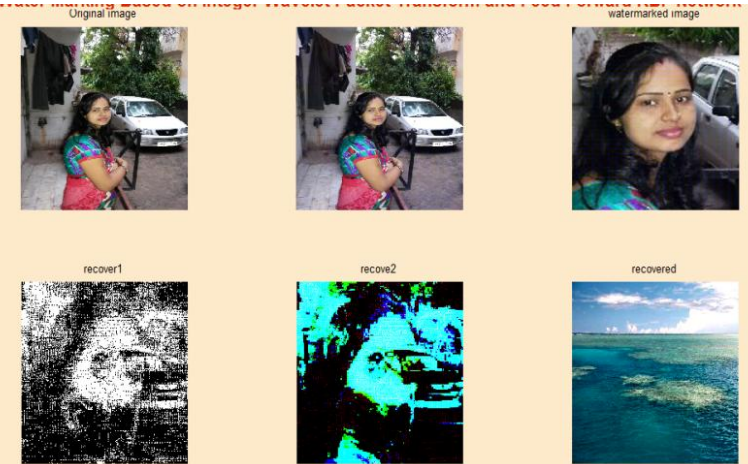

Figure 5 indicated that there were no visual differences between host image and the image embedded Watermark. And the watermarking had good imperceptibility. The peak signal to noise ratio for noise feature is PSNR $=23.70$, the extracted watermark and the original watermarking similarity was $\mathrm{NC}$ $=0.4424$.

\begin{tabular}{|c|c|c|c|c|c|}
\hline $\begin{array}{l}\text { Digital } \\
\text { Image }\end{array}$ & $\begin{array}{l}\text { Method of } \\
\text { Watermarkin } \\
g\end{array}$ & $\begin{array}{l}\text { Types of } \\
\text { Attack }\end{array}$ & $\begin{array}{l}\text { PSN } \\
\mathrm{R}\end{array}$ & $\mathrm{NC}$ & $\begin{array}{l}\text { Recove } \\
r \text { time }\end{array}$ \\
\hline \multirow{6}{*}{$\begin{array}{l}\text { LENA } \\
\text { IMAG } \\
\text { E }\end{array}$} & \multirow{3}{*}{ DWT } & $\begin{array}{l}\text { Croppin } \\
\mathrm{g}\end{array}$ & 21.04 & $\begin{array}{l}441 \\
5\end{array}$ & 17.25 \\
\hline & & Shear & 20.60 & $\begin{array}{l}.440 \\
2\end{array}$ & 12.33 \\
\hline & & Noise & 20.17 & $\begin{array}{l}.383 \\
2\end{array}$ & 10.42 \\
\hline & \multirow{3}{*}{ WRBF } & $\begin{array}{l}\text { Croppin } \\
\mathrm{g}\end{array}$ & 22.84 & $\begin{array}{l}.619 \\
0\end{array}$ & 3.58 \\
\hline & & Shear & 23.05 & $\begin{array}{l}.643 \\
1\end{array}$ & 2.69 \\
\hline & & Noise & 22.60 & $\begin{array}{l}.618 \\
8\end{array}$ & 4.05 \\
\hline
\end{tabular}

Table 1 shows the comparative PSNR, NC and Recover Time for Lena image for Digital image Watermarking on the basis of two methods DWT and WBRF.

\begin{tabular}{|c|c|c|c|c|c|}
\hline $\begin{array}{l}\text { Digital } \\
\text { Image }\end{array}$ & $\begin{array}{l}\text { Method of } \\
\text { Watermarkin } \\
\mathrm{g}\end{array}$ & $\begin{array}{l}\text { Types of } \\
\text { Attack }\end{array}$ & $\begin{array}{l}\text { PSN } \\
\mathrm{R}\end{array}$ & $\mathrm{NC}$ & $\begin{array}{l}\text { Recove } \\
\text { r time }\end{array}$ \\
\hline \multirow[b]{3}{*}{ BABY } & \multirow{3}{*}{ DWT } & $\begin{array}{l}\text { Croppin } \\
\mathrm{g}\end{array}$ & 22.53 & $\begin{array}{l}.187 \\
2\end{array}$ & 13.80 \\
\hline & & Shear & 22.34 & $\begin{array}{l}.283 \\
0\end{array}$ & 11.23 \\
\hline & & Noise & 22.03 & .242 & 9.84 \\
\hline
\end{tabular}

\begin{tabular}{|l|l|l|l|l|l|}
\hline \multirow{4}{*}{$\begin{array}{l}\text { IMAG } \\
\text { E }\end{array}$} & & & & 3 & \\
\cline { 3 - 6 } & \multirow{3}{*}{ WRBF } & $\begin{array}{l}\text { Croppin } \\
\mathrm{g}\end{array}$ & 25.00 & $\begin{array}{l}.365 \\
1\end{array}$ & 3.74 \\
& & Shear & 24.80 & $\begin{array}{l}.481 \\
5\end{array}$ & 3.18 \\
& & & 5 & \\
\cline { 3 - 6 } & & Noise & 24.48 & $\begin{array}{l}.474 \\
8\end{array}$ & 2.16 \\
& & & 8 & \\
\hline
\end{tabular}

Table 2 shows the comparative PSNR, NC and Recover Time for Baby image for Digital image Watermarking on the basis of two methods DWT and WBRF.

\begin{tabular}{|c|c|c|c|c|c|}
\hline $\begin{array}{c}\text { Digital } \\
\text { Image }\end{array}$ & $\begin{array}{c}\text { Method of } \\
\text { Watermarking }\end{array}$ & $\begin{array}{c}\text { Types of } \\
\text { Attack }\end{array}$ & PSNR & NC & $\begin{array}{c}\text { Recover } \\
\text { time }\end{array}$ \\
\hline \multirow{3}{*}{ FAMILY } & \multirow{3}{*}{ DWT } & Cropping & 20.65 & .5370 & 12.48 \\
\cline { 3 - 6 } & & Shear & 21.79 & .2934 & 12.54 \\
\cline { 3 - 6 } & & Noise & 21.25 & .2783 & 10.26 \\
\cline { 3 - 6 } & \multirow{3}{*}{ WRBF } & Cropping & 23.61 & .4238 & 3.54 \\
\cline { 3 - 6 } & & Shear & 24.24 & .4842 & 3.12 \\
\cline { 3 - 6 } & & Noise & 23.70 & .4424 & 3.83 \\
\hline
\end{tabular}

Table 3 shows the comparative PSNR, NC and Recover Time for Family image for Digital image Watermarking on the basis of two methods DWT and WBRF.

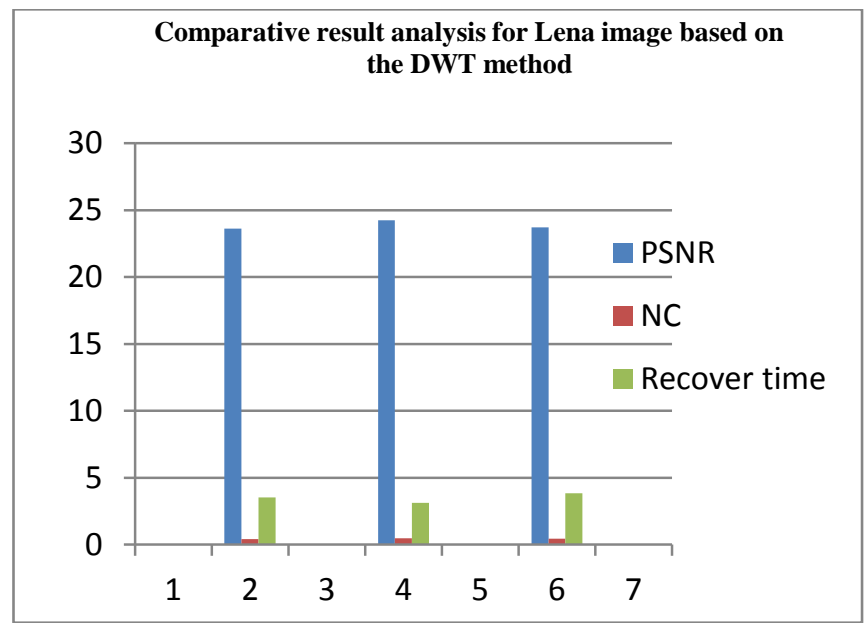

Figure 6 Shows that a comparative result analysis for Lena image based on the DWT method. We find the value of PSNR, $\mathrm{NC}$ and Recover time. These all value find with the some feture such as Cropping, Shear and Noise. 


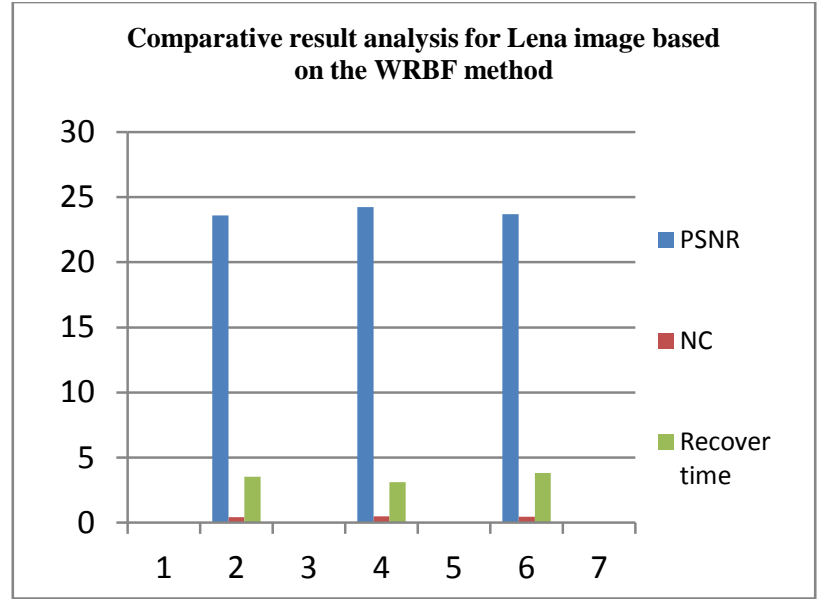

Figure 7 Shows that a comparative result analysis for Lena image based on the WRBF method. We find the value of

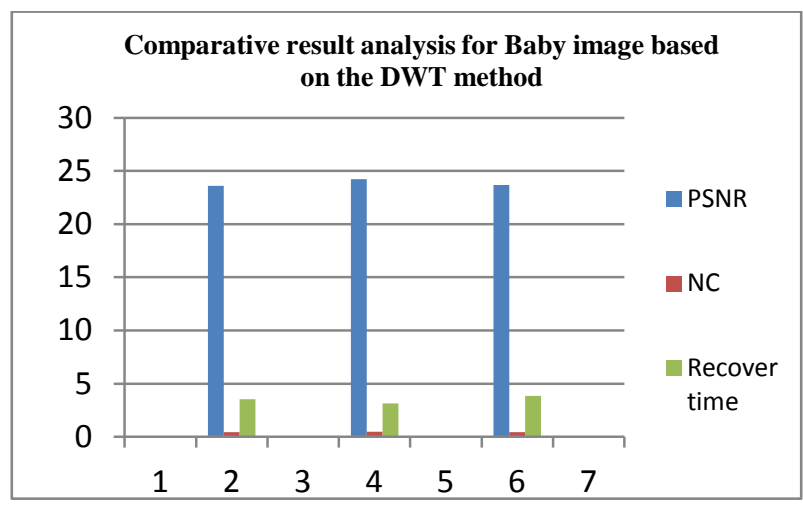

Figure 8 Shows that a comparative result analysis for Baby image based on the DWT method. We find the value of PSNR, $\mathrm{NC}$ and Recover time. These all value find with the some feture such as Cropping, Shear and Noise.

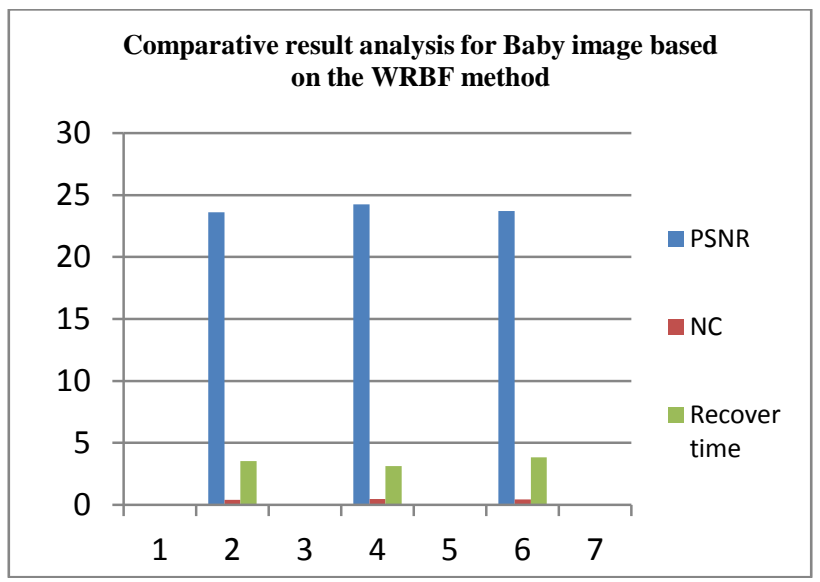

Figure 9 Shows that a comparative result analysis for Baby image based on the WRBF method. We find the value of PSNR, NC and Recover time. These all value find with the some feture such as Cropping, Shear and Noise.

\section{CONCLUSION AND FUTURE SCOPE}

In this paper proposed a novel method for digital water marking technique based on feature attribute selection based using ID3 and RBF function. The feature extraction process used integer wavelet transform function and extracted feature passes through ID3 algorithm for find appropriate attribute and after that RBF function applied for embedding. The proposed method simulates MATLAB software. For the test of roughness used some standard attack such as noise attack, shear attack and translation attack. We find the better performance in compression of DWT watermarking technique. The process of watermarking technique is very complex so computational time is increase. Now in future reduce the computational time of water marking technique.

\section{REFERENCES}

[1] Chih-ChinLai, Chi-Feng Chan, Chen-Sen Ouyang, Hui-Fen Chiang "A Robust Feature-based Image Watermarking Scheme" 14th ACIS International Conference on Software Engineering, Artificial Intelligence, Networking and Parallel/Distributed Computing, IEEE 2013. Pp 581-586.

[2] B.Jagadeesh, P.Rajesh Kumar, P.Chenna Reddy "Robust Digital Image Watermarking Scheme in Discrete Wavelet Transform domain using Support Vector Machines" International Journal of Computer Applications" Volume 73, 2013. Pp 1-8.

[3] B.Sridhar, Dr.C.Arun" A Wavelet based Image Watermarking Technique using Image Sharing Method" IEEE, 2012. Pp 1-5.

[4] Qiao Baoming, Zhang Pulin, Kang Qiao "A Digital Watermarking Algorithm Based on Wavelet Packet Transform and BP Neural Network" Seventh International Conference on Computational Intelligence and Security, IEEE 2011. Pp 503-507.

[5] R. Ni, Q. Ruan, and H. D. Cheng "Secure semi-blind watermarking based on iteration mapping and image features" Pattern Recognition, vol. 38, no. 3, 2005. Pp $357-368$

[6] C.-W. Tang and H.-M. Hang "A feature-based robust digital image watermarking scheme" IEEE Trans. Signal Processing, vol. 51, no. 4,2003. Pp 950-959.

[7] J. S. Seo and C. D. Yoo "Localized image watermarking based on feature points of scale-space representation" Pattern Recognition, vol. 37, no. 7,2004. Pp 1365-1375.

[8] X. Qi and J. Qi, "A robust content-based digital image watermarking scheme," Signal Processing, vol. 87, no. 6, 2007 Pp 1264-1280.

[9] X.-y. Wang, L.-m. Hou, and J.Wu "A feature-based robust digital image watermarking against geometric attack" Image and Vision Computing, vol. 26, no. 7, 2008. Pp 980-989.

[10] Fu, Y, Shen, R, Lu, H "Optimal watermark Detection based on support vector machines" Proceedings of the International Symposium on Neural Networks, Dalian, China,2004. Pp 552-557.

[11] H.H. Tsaia, H.C. Tsenga, Y.S. Laib "Robust lossless image watermarking based on $\alpha$-trimmed mean algorithm and support vector machine", J. Sys. Software. 83 (6), 2007. Pp 1015 -1028. 
[12] Chun-hua Li, Ling He-fei, Lu Zheng-ding "Semi-fragile watermarking based on SVM for image authentication", IEEE International Conference on Multimedia and Expo, Beijing, China, 2007, Pp 1255-1258.

[13] Hang SU, Chuqing LV, Yanbing JI, Yulin WANG “A Watermarking E-note Technique against Geometric Attacks" 2nd International Conference on Mechanical and Electronics Engineering, IEEE 2010. Pp 446-448.
[14] Xiaolin Li, Yan Feng. "Image rotation policy based on DSP althorithm" The Application of Power Electronics, 2008.

[15] Heena Shaikh,Mohd.Imran Khan, Yashovardhan Kelkar "A Robust DWT Digital Image Watermarking Technique Basis On Scaling Factor" International Journal of Computer Science, Engineering and Applications, Vol-2, 2012. Pp 63-70.

[16] Liu Yuxun and Xie Niuniu "Improved ID3 Algorithm" in IEEE, 2010 